\title{
Fourier Method in Initial Boundary Value Problems for Regions with Curvilinear Boundaries
}

\author{
Leontiev V. L. \\ Institute of Advanced Manufacturing Technologies at Peter the Great St. Petersburg Polytechnic University, Russia
}

Received October 28, 2020; Revised December 29, 2020; Accepted January 28, 2021

\section{Cite This Paper in the following Citation Styles}

(a): [1] Leontiev V. L. , "Fourier Method in Initial Boundary Value Problems for Regions with Curvilinear Boundaries," Mathematics and Statistics, Vol. 9, No. 1, pp. 24-30, 2021. DOI: 10.13189/ms.2021.090104.

(b): Leontiev V. L. (2021). Fourier Method in Initial Boundary Value Problems for Regions with Curvilinear Boundaries. Mathematics and Statistics, 9(1), 24-30. DOI: 10.13189/ms.2021.090104.

Copyright $\mathrm{C} 2021$ by authors, all rights reserved. Authors agree that this article remains permanently open access under the terms of the Creative Commons Attribution License 4.0 International License

\begin{abstract}
The algorithm of the generalized Fourier method associated with the use of orthogonal splines is presented on the example of an initial boundary value problem for a region with a curvilinear boundary. It is shown that the sequence of finite Fourier series formed by the method algorithm converges at each moment to the exact solution of the problem - an infinite Fourier series. The structure of these finite Fourier series is similar to that of partial sums of an infinite Fourier series. As the number of grid nodes increases in the area under consideration with a curvilinear boundary, the approximate eigenvalues and eigenfunctions of the boundary value problem converge to the exact eigenvalues and eigenfunctions, and the finite Fourier series approach the exact solution of the initial boundary value problem. The method provides arbitrarily accurate approximate analytical solutions to the problem, similar in structure to the exact solution, and therefore belongs to the group of analytical methods for constructing solutions in the form of orthogonal series. The obtained theoretical results are confirmed by the results of solving a test problem for which both the exact solution and analytical solutions of discrete problems for any number of grid nodes are known. The solution of test problem confirm the findings of the theoretical study of the convergence of the proposed method and the proposed algorithm of the method of separation of variables associated with orthogonal splines, yields the approximate analytical solutions of initial boundary value problem in the form of a finite Fourier series with any desired accuracy. For any number of grid nodes, the method leads to a generalized finite Fourier series which corresponds with high accuracy to the partial sum of the Fourier series of the exact solution of the problem.
\end{abstract}

Keywords Initial Boundary Value Problems, Curvilinear Boundary, Separation Variable Method, Fourier Series, Orthogonal Splines

\section{Introduction}

\section{Problem Statement}

The method of separation of variables (Fourier method) allows finding partial solutions of many boundary value and initial boundary value problems for partial differential equations that allow separation of variables. The method is related to the Sturm-Liouville problem and, in many cases, to special functions at the stage of solving this problem. The classical Fourier method allows obtaining solutions to a wide class of problems, but its implementation for many types of problems, including problems whose statements contain irregular boundary conditions, even in cases where all parts of the boundary of the region are coordinate lines or surfaces, meets with significant difficulties. One of the ways to expand the scope of the classical Fourier method is to solve mathematical problems related to the nature of boundary conditions [1]. Special functions appear in the algorithm of the method when solving the Sturm-Liouville problem in cylindrical or spherical coordinate systems in cases of regions whose boundaries are coordinate lines or surfaces. In the general case of problems for areas with curvilinear boundaries, the use of special functions is inefficient. The classical Fourier method is applicable only for solving 
boundary value and initial boundary value problems for regions of classical shape, which is noted, for example, in solving contact problems [2] for elastic bodies with curvilinear boundaries. The solutions obtained by the classical Fourier method are given, in particular, in the articles $[3,4]$, the application of the method is considered in many books, for example, in [5]. Other areas of development of mathematical tools for solving problems for areas with curvilinear boundaries are associated, first, with the creation and application of a number of methods other than the Fourier method, for example [2, 6, 7, 8], and, secondly, with a modification of the Fourier method itself. This article aims to expand the scope of the classical Fourier method, defined by the application of a sequence of finite generalized Fourier series and orthogonal splines.

The next first initial boundary value problem is considered

$$
\begin{aligned}
& L[u]=a^{2} \Delta u(x, y, t)=a^{2}\left(\frac{\partial^{2} u}{\partial x^{2}}+\frac{\partial^{2} u}{\partial y^{2}}\right)=\frac{\partial^{2} u}{\partial t^{2}} \\
& \forall(x, y) \in S, \forall t \geq 0 ; \quad u(x, y, 0)=f(x, y), \\
& \left.\frac{\partial u(x, y, t)}{\partial t}\right|_{t=0}=F(x, y) \quad \forall(x, y) \in S ; \\
& \left.u\right|_{\partial S}=0 \quad \forall t \geq 0 ;
\end{aligned}
$$

where $\partial S$ is a piecewise smooth curvilinear boundary of the region $S, u=u(x, y, t)$ - a function, continuous $\forall t \geq 0$ in a closed area $\bar{S}=S+\partial S, a^{2}=$ const $>0$.

According to the method of separating variables, the solution of problem (1) is sought as a product of two functions

$$
u(x, y, t)=\varphi(x, y) \cdot \psi(t),
$$

substituting which in the differential equation (1) leads to

$$
L[\varphi(x, y)] \cdot \psi(t)=\varphi(x, y) \partial^{2} \psi(t) / \partial t^{2}
$$

or

$$
\begin{gathered}
\frac{L[\varphi(x, y)]}{\varphi(x, y)}=\frac{\partial^{2} \psi(t) / \partial t^{2}}{\psi(t)}= \\
=-\lambda=\operatorname{const}(x, y, t)<0 .
\end{gathered}
$$

The boundary condition (1) taking into account (2) gives

$$
\left.\varphi(x, y)\right|_{\partial S}=0
$$

so from (3) follows the Sturm-Liouville boundary value problem

$$
\begin{aligned}
& L[\varphi]+\lambda \varphi=a^{2} \Delta \varphi+\lambda \varphi=0 \quad(S), \\
& \left.\varphi\right|_{\partial S}=0,
\end{aligned}
$$

intended for defining a function $\varphi(x, y)$. After solving this problem, that is, after constructing systems of eigenvalues and corresponding eigenfunctions $\varphi_{k}(x, y)$, the corresponding system of solutions $\psi_{k}(t)$ of the equation

$$
\partial^{2} \psi(t) / \partial t^{2}+\lambda \psi(t)=0
$$

is found. Then, based on $\psi_{k}(t)$ and $\varphi_{k}(x, y)$ taking into account the initial conditions, an infinite Fourier series is constructed - the exact solution of the problem (1).

\section{The Algorithm of the Method}

The first steps of the generalized Fourier method algorithm for regions with a curvilinear boundary coincide with similar steps of the classical Fourier method. The solution of problem (1) is also sought in the form of a product (2), whose substitution in the differential equation (1) leads to equation (3), and the boundary condition (1) gives (4). The same Sturm-Liouville boundary value problem (5) arises. From (3) also follows equation (6), the solution of which is related to the solution of the Sturm-Liouville problem (5) and taking into account two initial conditions (1).

The further steps of the algorithm of the generalized Fourier method, designed for solving initial boundary value problems in the case of regions with curvilinear boundaries, differ from the corresponding steps of the classical algorithm. They are related to the application of orthogonal splines in the construction of a sequence of approximate analytical solutions in the form of finite Fourier series.

Nontrivial solutions of the boundary value problem (5) are sought in the form

$$
\varphi_{N}(x, y)=\sum_{i=1}^{N} \sum_{j=j_{1}(i)}^{j_{2}(i)} \widetilde{c}_{i j} \widetilde{\alpha}_{i}(x) \widetilde{\beta}_{j}(y),
$$

where $\tilde{c}_{i j}$ are constant coefficients; $N, j_{1}, j_{2}-$ natural numbers; the dependence $j_{1}, j_{2}$ on $i$ is determined by a curvilinear boundary $\partial S ; \quad \widetilde{\alpha}_{i}(x), \quad \widetilde{\beta}_{j}(y)$ - continuous orthogonal splines $[9,10]$ :

$$
\tilde{\alpha}_{i}(x)=\varphi_{i}(x), \quad \tilde{\beta}_{j}(y)=\varphi_{j}(y) .
$$

The orthogonal splines $\varphi_{i}(x)$ in the case of a rectangular uniform grid have the form [9] 


$$
\varphi_{i}(x)=\left\{\begin{array}{c}
(\sqrt{2}-1)\left(x_{i-1}-x\right) / h, x \in\left[x_{i-1}, x_{i-1}+\frac{h}{2}\right] \\
(\sqrt{2}+1)\left(x-x_{i}\right) / h+1, x \in\left[x_{i-1}+\frac{h}{2}, x_{i}\right] \\
(\sqrt{2}-1)\left(x-x_{i}\right) / h+1, x \in\left[x_{i}, x_{i}+\frac{h}{2},\right] \\
(\sqrt{2}+1)\left(x_{i+1}-x\right) / h, x \in\left[x_{i}+\frac{h}{2}, x_{i+1}\right] \\
0, \quad x \in\left(-\infty, x_{i-1}\right) \cup\left(x_{i+1},+\infty\right)
\end{array}\right.
$$

where $h_{1}=h_{2}=h-$ steps of grid, the nodes of a grid have coordinates

$$
\left(x_{i}=i h, \quad y_{j}=j h\right) \in \bar{S}, 1 \leq i \leq N, 1 \leq j \leq N .
$$

Scalar products have the properties

$$
\left(\widetilde{\alpha}_{i}, \widetilde{\alpha}_{j}\right)=\left\|\widetilde{\alpha}_{i}\right\|^{2} \delta_{i j}, \quad\left(\widetilde{\beta}_{i}, \widetilde{\beta}_{j}\right)=\left\|\widetilde{\beta}_{i}\right\|^{2} \delta_{i j},
$$

where $\delta_{i j}$ are the Kronecker symbols. The region $S$ with a curvilinear boundary $\partial S$ is inscribed in a rectangle $S_{1}=\{A \leq x \leq B ; C \leq y \leq D\}$. The compact supports of tensor products of orthogonal splines on each grid are rectangular subdomains. Orthogonal splines also allow the use of triangular compact supports. The functions

$$
\begin{aligned}
& \alpha_{i}(x)=\widetilde{\alpha}_{i}(x) /\left\|\widetilde{\alpha}_{i}(x)\right\|, \\
& \beta_{j}(y)=\widetilde{\beta}_{j}(y) /\left\|\widetilde{\beta}_{j}(y)\right\|
\end{aligned}
$$

form two systems of orthonormal splines. The sum (7) is rewritten as

$$
\varphi_{N}(x, y)=\sum_{i=1}^{N} \sum_{j=j_{1}(i)}^{j_{2}(i)} c_{i j} \alpha_{i}(x) \beta_{j}(y),
$$

where each constant coefficient $c_{i j}$ is equal to a value of $\varphi_{N}(x, y)$ at the node $\left(x_{i}, y_{j}\right)$ of a grid. Therefore, the boundary conditions $\left.\varphi\right|_{\partial S}=0$ are satisfied if the coefficients $c_{i j}$, corresponding to the nodes associated with the boundary $\partial S$, are equal to zero. The sum (8), taking into account the boundary conditions, takes the form

$$
\varphi_{N}(x, y)=\sum_{i=N_{1}}^{N_{2}} \sum_{j=j_{1}^{*}(i)}^{j_{2}^{*}(i)} c_{i j} \alpha_{i}(x) \beta_{j}(y),
$$

where

$$
N_{1}, N_{2}, j_{1}^{*}, j_{2}^{*}: 1 \leq N_{1}<N_{2} \leq N ; j_{1} \leq j_{1}^{*}<j_{2}^{*} \leq j_{2} .
$$

To determine the coefficients $c_{i j}$ of the approximate analytical solution (9) of the boundary value problem (5), the Reissner variational principle is used: $\delta R=0$, where

$$
\begin{aligned}
2 R\left(\varphi, \varphi_{1}, \varphi_{2}\right) & =\int_{S}\left\{\left[a^{2}\left(\partial \varphi_{1} / \partial x+\partial \varphi_{2} / \partial y\right)+\lambda \varphi\right] \varphi-\right. \\
-(\partial \varphi / \partial x & \left.\left.-\varphi_{1}\right) \varphi_{1}-\left(\partial \varphi / \partial y-\varphi_{2}\right) \varphi_{2}\right\} d S+ \\
& +\int_{\partial S} \varphi\left(\varphi_{1} n_{x}+\varphi_{2} n_{y}\right) d l
\end{aligned}
$$

where $n_{x}, n_{y}-$ components of normal to a boundary $\partial S$. The variational principle is equivalent to problem (5) in mixed form

$$
\begin{gathered}
a^{2}\left(\partial \varphi_{1} / \partial x+\partial \varphi_{2} / \partial y\right)+\lambda \varphi=0, \partial \varphi / \partial x=\varphi_{1}, \\
\partial \varphi / \partial y=\varphi_{2} \quad(S) ;\left.\quad \varphi\right|_{\partial S}=0,
\end{gathered}
$$

and after substituting (9) into $\delta R=0$, it leads to a system of finite difference equations [10] for the internal nodes of the grid

$$
\begin{gathered}
a^{2}\left[\left(\varphi_{n+1, m}-2 \varphi_{n m}+\varphi_{n-1, m}\right) / h_{1}^{2}+\left(\varphi_{n, m+1}-\right.\right. \\
\left.\left.-2 \varphi_{n m}+\varphi_{n, m-1}\right) / h_{2}^{2}\right]+\lambda_{n m} \varphi_{n m}=0 .
\end{gathered}
$$

After taking into account the boundary condition $\varphi_{n m}=0$ for nodes that fall on the boundary $\partial S$ of the region $S$, a homogeneous system of linear algebraic equations is formed, in which the coefficients $c_{i j}$ are unknown. Those values $\lambda_{k}=\lambda_{n m}$, for which system (10) has nontrivial solutions are the eigenvalues of the real and symmetric matrix of system (10). All eigenvalues $\lambda_{k}(k=1,2, \ldots, K)$ and their corresponding eigenvectors $X^{(k)}$ of this matrix have real values, and all eigenvectors are linearly independent and pairwise orthogonal, including in the case when there are multiple matching eigenvalues.

\section{Theorem 1. The function}

$$
\begin{gathered}
u^{(K)}(x, y, t)=\sum_{k=1}^{K}\left[\left(A_{k} \cos \sqrt{\lambda_{k}} t+\right.\right. \\
\left.\left.+B_{k} \sin \sqrt{\lambda_{k}} t\right) \sum_{i=N_{1}}^{N_{2}} \sum_{j=j_{1}^{*}(i)}^{j_{2}^{*}(i)} C_{i j}^{(k)} \alpha_{i}(x) \beta_{j}(y)\right],
\end{gathered}
$$

where

$$
A_{k}=\frac{1}{\left\|\varphi_{N}^{(k)}\right\|^{2}} \iint_{S} f(x, y) \varphi_{N}^{(k)}(x, y) d S,
$$

$$
B_{k}=\frac{1}{\sqrt{\lambda_{k}}\left\|\varphi_{N}^{(k)}\right\|^{2}} \iint_{S} F(x, y) \varphi_{N}^{(k)}(x, y) d S,
$$

satisfies equations (5) in variational form, equation (6), as well as the boundary condition and two initial conditions 
(1), so, it is an approximate analytical solution of problem (1) in the case of a region $S$ with a curvilinear boundary $\partial S$. For each fixed time value, this sum is a finite generalized Fourier series over the eigenfunctions of the boundary value problem (5) in variational form.

Proof. The sum

$$
\varphi_{N}^{(k)}(x, y)=\sum_{i=N_{1}}^{N_{2}} \sum_{j=j_{1}^{*}(i)}^{j_{2}^{*}(i)} C_{i j}^{(k)} \alpha_{i}(x) \beta_{j}(y)
$$

is an element of the set $H_{N}$ - a linear shell of functions $\alpha_{i}(x) \beta_{j}(y)$, associated with the specified grid and satisfying the boundary condition. This sum is also a nontrivial solution of the system of equations (10) that satisfies the boundary condition (5) and corresponds to the eigenvalue $\lambda_{k}$, that is, $\varphi_{N}^{(k)}(x, y)$ - the eigenfunction of the boundary value problem (5) in the variational form.

Consider equation (6) after substituting any found positive eigenvalue into it

$$
\partial^{2} \psi(t) / \partial t^{2}+\lambda_{k} \psi(t)=0
$$
form

The general solution of this differential equation has the

$$
\psi_{k}(t)=A_{k} \cos \sqrt{\lambda_{k}} t+B_{k} \sin \sqrt{\lambda_{k}} t,
$$

where $A_{k}, B_{k}$ are unknown constant coefficients.

Therefore, the sum

$$
\begin{aligned}
& \sum_{k=1}^{K} \psi_{k}(t) \varphi_{N}^{(k)}(x, y)=\sum_{k=1}^{K}\left[\left(A_{k} \cos \sqrt{\lambda_{k}} t+\right.\right. \\
& \left.\left.+B_{k} \sin \sqrt{\lambda_{k}} t\right) \sum_{i=N_{1}}^{N_{2}} \sum_{j=j_{1}^{*}(i)}^{j_{2}^{*}(i)} C_{i j}^{(k)} \alpha_{i}(x) \beta_{j}(y)\right]
\end{aligned}
$$

satisfies equation (5) in variational form, equation (6), and boundary condition (5). It remains to satisfy the initial conditions. The substitution of the sum (14) into the first initial condition (1) gives

$$
\sum_{k=1}^{K} A_{k} \varphi_{N}^{(k)}(x, y)=f(x, y) .
$$

Multiplying both parts of the last equation by $\varphi_{N}^{(r)}(x, y)$, integrating over the region $S$ and using the orthogonality of the eigenfunctions allow writing

$$
A_{k}=\frac{1}{\left\|\varphi_{N}^{(k)}\right\|^{2}} \iint_{S} f(x, y) \varphi_{N}^{(k)}(x, y) d S .
$$

Substituting the sum (14) in the second initial condition (1) gives

$$
\sum_{k=1}^{K} \sqrt{\lambda_{k}} B_{k} \varphi_{N}^{(k)}(x, y)=F(x, y) .
$$

Multiplying both parts of this equation by $\varphi_{N}^{(r)}(x, y)$, integrating over the region $S$ allow writing

$$
B_{k}=\frac{1}{\sqrt{\lambda_{k}}\left\|\varphi_{N}^{(k)}\right\|^{2}} \iint_{S} F(x, y) \varphi_{N}^{(k)}(x, y) d S .
$$

Thus, the sum (11), whose coefficients are determined by formulas (12), (13), satisfies equation (5) in the variational form, equation (6), as well as the boundary condition (5) and two initial conditions (1), that is, the sum (11) is an approximate analytical solution of the problem (1) in the case of a region with a curvilinear boundary. The sum (11) for each fixed time value is a finite generalized Fourier series over the eigenfunctions of the boundary value problem in variational form.

The proof is completed.

\section{The Convergence of the Method}

Theorem 2. The approximate eigenfunctions $\varphi_{N}^{(k)}(x, y)$ converge to exact eigenfunctions $\varphi^{(k)}(x, y) \in W_{2}^{1}(\bar{S})$, satisfying the boundary condition (5), that is,

$$
\left\|\varphi^{(k)}-\varphi_{N}^{(k)}\right\|_{W_{2}^{1}(S)}^{2}->0 \quad \text { if } \quad N \rightarrow \infty .
$$

Here $W_{2}^{1}(\bar{S})$ is Sobolev space.

Proof. The condition of stationary $\delta \Phi=0$ of the functional

$$
\Phi\left(\varphi^{(k)}\right)=\iint_{S}\left[a^{2}\left(\bar{\nabla} \varphi^{(k)}\right)^{2}-\lambda_{k}\left(\varphi^{(k)}\right)^{2}\right] d S,
$$

is equivalent to the boundary value problem (5). Here $\bar{\nabla}$ is the nabla operator. The variation of the functional is carried out on the set of functions satisfying the main boundary condition (5). The value of the second variation of the functional at its stationary point is positive, and therefore the functional has a minimum at the stationary point, and the solution of the variational problem, and hence the boundary value problem (5), are unique. In addition, the functional has a value equal to zero at a stationary point $\varphi^{(k)}$, since on the set of functions $W_{2}^{1}(\bar{S})$, satisfying the boundary condition,

$$
\Phi\left(\varphi^{(k)}\right)=-\iint_{S}\left[a^{2} \Delta \varphi^{(k)}+\lambda_{k} \varphi^{(k)}\right] \varphi^{(k)} d S=0 .
$$

The functional for any function $w$ of the set $W_{2}^{1}(\bar{S})$, 
that satisfies the boundary condition is

$$
\begin{gathered}
\Phi(w)=\iint_{S}\left[a^{2}(\bar{\nabla} w)^{2}-\lambda_{k} w^{2}\right] d S= \\
=\iint_{S}\left[a^{2}\left(\bar{\nabla}\left(\varphi^{(k)}-w\right)\right)^{2}-\lambda_{k}\left(\varphi^{(k)}-w\right)^{2}\right] d S= \\
=\left[\varphi^{(k)}-w, \varphi^{(k)}-w\right]-\lambda_{k}\left(\varphi^{(k)}-w, \varphi^{(k)}-w\right)
\end{gathered}
$$

- the difference of the energy scalar product

$$
\left[v_{1}, v_{2}\right]=\iint_{S} a^{2}\left(\bar{\nabla} v_{1}\right) \cdot \bar{\nabla} v_{2} d S
$$

and scalar product

$$
\left(v_{1}, v_{2}\right)=\iint_{S} v_{1} v_{2} d S .
$$

Thus, the problem of finding the exact eigenfunctions $\varphi^{(k)}$ was reduced to the problem of minimization

$$
\Phi\left(\varphi^{(k)}\right)=\min _{\forall w \in W_{2}^{1}(\bar{S})} \Phi(w)=0
$$

at the set of functions $w$, satisfying the boundary condition. Because

$$
\begin{gathered}
|\Phi(w)|=\mid\left[\varphi^{(k)}-w, \varphi^{(k)}-w\right]- \\
-\lambda_{k}\left(\varphi^{(k)}-w, \varphi^{(k)}-w\right) \mid \leq \\
\leq\left|\left[\varphi^{(k)}-w, \varphi^{(k)}-w\right]\right|+\lambda_{k}\left|\left(\varphi^{(k)}-w, \varphi^{(k)}-w\right)\right|,
\end{gathered}
$$

then the minimization problem (14) is reduced to the approximation theory problem

$$
\left\|\varphi^{(k)}-\varphi_{N}^{(k)}\right\|_{W_{2}^{1}(S)}^{2}=\min _{\forall w \in H_{N} \subset W_{2}^{1}(\bar{S})}\left\|\varphi^{(k)}-w\right\|_{W_{2}^{1}(S)}^{2},
$$

that is, to the problem of approximation of exact eigenfunctions $\varphi^{(k)}(x, y)$ by linear combinations $\varphi_{N}^{(k)}(x, y)$ of orthogonal splines. This problem is solved in $[9,10]$, there are corresponding approximation theorems that determine the accuracy of the approximation and the rate of convergence, depending on the type of orthogonal splines. The proof is completed.

When the number of grid nodes in the region $S$ increases, i.e., when the number of orthogonal splines used increases, the eigenvalues of the system of difference equations (10) in the case $l=\pi$ have the form $[9,10]$

$$
\begin{gathered}
\lambda_{k}=\lambda_{n m}=n^{2}+m^{2}+ \\
+\left(n^{4}+m^{4}\right) h^{2} / 12+O\left[\left(n^{6}+m^{6}\right) h^{4}\right]
\end{gathered}
$$

and converge to the corresponding exact eigenvalues

$$
\omega_{n m}=\pi^{2}\left(n^{2}+m^{2}\right) / l^{2}=n^{2}+m^{2}
$$

of the boundary value problem (5). As the number of grid nodes in the region $S$ increases, the approximate solutions $\varphi_{N}^{(k)}(x, y)$ of the boundary value problem (5), i.e. the approximate eigenfunctions of this problem, converge to its exact solutions - eigenfunctions $\varphi^{(k)}(x, y)$. In this case, the number of eigenvalues and eigenfunctions of the boundary value problem in variational form increases indefinitely, and consequently, the finite sum in the limit passes into an infinite series, which for any value $t>0$ is an infinite Fourier series. Such a series is the unique solution of problem (1), it follows from Steklov's theorem [11].

The difference between this method for solving initial boundary value problems for regions with curvilinear boundaries and other methods, for example, the finite element method [8], is that in this method, the sequence of finite Fourier series (11) determined by its algorithm converges at each fixed time to the corresponding infinite Fourier series formed on the basis of exact eigenfunctions $\varphi^{(k)}(x, y)$ and representing an existing exact solution to the problem (1), which cannot be determined. Consequently, these finite Fourier series represent analytical approximate solutions of problem (1) for a region with a curvilinear boundary, which, as the number of grid nodes increases, come close to the exact solution of this problem - an infinite Fourier series, not only in terms of quantitative criteria, but also in their analytical structure. The method gives, in the form of finite Fourier series, arbitrarily accurate approximate analytical solutions to problem (1) for regions with a curvilinear boundary whose structures are similar to the structure of the exact solution, and in this sense the method leads to an exact analytical solution after the required accuracy of the desired solution is set.

\section{Test Problem}

The problem (1) and the Sturm-Liouville problem (5) are considered for a region $\bar{S}$ whose boundary $\partial S$ is a square with a side $l=\pi$. A rectangular uniform grid with steps $h_{1}=h_{2}=h$ is used, the nodes of which have coordinates

$$
\left(x_{i}=i h, \quad y_{j}=j h\right) \in \bar{S}, \quad 0 \leq i \leq N, 0 \leq j \leq N .
$$

The finite difference equations (10) written for all internal nodes $1 \leq i \leq N-1,1 \leq j \leq N-1$, taking into account the boundary conditions (5), give a homogeneous system of difference equations, the nontrivial solutions of which are the eigenfunctions [12],[13],[14]

$$
\begin{gathered}
\mu_{n m}(i, j)=\sin (n \pi i / N) \sin (m \pi j / N)= \\
=\sin \left(n \pi x_{i} / l\right) \sin \left(m \pi y_{j} / l\right),
\end{gathered}
$$


corresponding to eigenvalues

$$
\begin{gathered}
\lambda_{n m}=4\left\{\sin ^{2}[n \pi h /(2 l)]+\sin ^{2}[m \pi h /(2 l)]\right\} / h^{2} . \\
n, m=1,2, \ldots, N-1 .
\end{gathered}
$$

In the case $l=\pi$ exact solutions of the system of finite difference equations have the form

$$
\begin{gathered}
\mu_{n m}(i, j)=\sin \left(n x_{i}\right) \sin \left(m y_{j}\right), \\
\lambda_{n m}=4\left[\sin ^{2}(n h / 2)+\sin ^{2}(m h / 2)\right] / h^{2} . \\
n, m=1,2, \ldots, N-1 .
\end{gathered}
$$

The number of eigenfunctions is equal $(N-1)^{2}-$ to the number of internal grid nodes. Any grid function $\varphi(i, j)$ defined on a grid of the area $\bar{S}$ can be represented as a linear combination of these eigenfunctions. The values $\mu_{n m}(i, j)$ of the eigenfunctions in the grid nodes, which correspond to $\lambda_{n m}$, together represent a nontrivial solution of the system of difference equations for each set $n, m$ or $k$. That is, $C_{i j}^{(k)}=\sin \left(n x_{i}\right) \sin \left(m y_{j}\right)$ and so in this problem

$$
\begin{aligned}
& \varphi_{N}^{(k)}(x, y)=\sum_{i=N_{1}}^{N_{2}} \sum_{j=j_{1}^{*}(i)}^{j_{2}^{*}(i)} C_{i j}^{(k)} \alpha_{i}(x) \beta_{j}(y)= \\
& =\sum_{i=1}^{N-1} \sum_{j=1}^{N-1} \sin \left(n x_{i}\right) \sin \left(m y_{j}\right) \alpha_{i}(x) \beta_{j}(y) .
\end{aligned}
$$

The finite series was formed for each $\lambda_{n m}$, the coefficients of it are equal to $\mu_{n m}(i, j)$.

Therefore, the finite series (11) has the form

$$
\begin{gathered}
u^{(N)}(x, y, t)=\sum_{n=1}^{N-1} \sum_{m=1}^{N-1}\left[\left(A_{n m} \cos \sqrt{\lambda_{n m}} t+\right.\right. \\
\left.+B_{n m} \sin \sqrt{\lambda_{n m}} t\right) \varphi_{N}^{(k)}(x, y) .
\end{gathered}
$$

Its coefficients $A_{k}=A_{n m}, B_{k}=B_{n m}$ are determined by formulas (12), (13), which take into account the initial conditions (1).

The exact solution of the Sturm-Liouville problem (5) for the case $a^{2}=1$ and for the case of the square domain $\bar{S}$ is determined by eigenfunctions and eigenvalues [11]:

$$
\begin{gathered}
\Phi_{n m}(x, y)=\sin (n \pi x / l) \sin (m \pi y / l), \\
\omega_{n m}=\pi^{2}\left(n^{2}+m^{2}\right) / l^{2}=n^{2}+m^{2} .
\end{gathered}
$$

Based on the exact solution of problem (5), the exact solution of problem (1) has the form [11]

$$
\begin{gathered}
u(x, y, t)=\sum_{n=1}^{\infty} \sum_{m=1}^{\infty}\left(C_{n m} \cos \sqrt{\omega_{n m}} t+\right. \\
\left.+D_{n m} \sin \sqrt{\omega_{n m}} t\right) \sin (n \pi x / l) \sin (m \pi y / l) .
\end{gathered}
$$

Its coefficients $C_{n m}, D_{n m}$ are determined by formulas

$$
\begin{gathered}
C_{n m}=\frac{1}{\left\|\Phi_{n m}\right\|^{2}} \iint_{S} f(x, y) \Phi_{n m}(x, y) d S, \\
D_{n m}=\frac{1}{\sqrt{\lambda_{k}}\left\|\Phi_{n m}\right\|^{2}} \iint_{S} F(x, y) \Phi_{n m}(x, y) d S,
\end{gathered}
$$

that take into account the initial conditions (1).

Accuracy of approximation of eigenfunctions $\Phi_{n m}(x, y)$ by approximate eigenfunctions

$$
\varphi_{N}^{(k)}(x, y)=\varphi_{N}^{(n m)}(x, y)
$$

is increased with increasing $N$ and the values $A_{n m}, B_{n m}$ converge accordingly with the values $C_{n m}$ and $D_{n m}$.

The finite generalized Fourier series (15) is characterized by the following. Orthogonal splines $\alpha_{i}(x), \beta_{j}(y)$ are continuous and piecewise linear, their values equal to unity in grid nodes, so the values at the grid points internal double sum $\varphi_{N}^{(k)}(x, y)$ equal to the value of eigenfunctions $\sin (n \pi x / l) \sin (m \pi y / l)$ at these nodes. In addition, all $\lambda_{n m} \rightarrow \omega_{n m}$, when $N \rightarrow \infty$. So, for example, $\lambda_{11}=1.899$ at $N=4$, $\lambda_{11}=1.984$ at $N=10, \lambda_{11}=1.996$ at $N=20$, etc., that is, $\lambda_{11}$ converges to the exact value $\omega_{11}=2$. Thus, as the number $N$ of eigenfunctions in $u^{(N)}(x, y, t)$ increases, the accuracy of the approximation of all these functions between nodes increases, and in nodes it is accurate for each value $N$. The number and accuracy of accounted eigenvalues also increases in $u^{(N)}(x, y, t)$. The structure of the finite series $u^{(N)}(x, y, t)$ corresponds to the structure of the partial sum of the infinite series (16), with account of the number of nodes for a concrete calculation. Thus, the formulations of theorems 1 and 2 are confirmed by the results of solving the test problem. These results confirm the conclusions of the theoretical study of the convergence of the method, and that the proposed algorithm of the method of separating variables, which is connected with orthogonal splines, gives approximate analytical solutions to the initial boundary value problem in the form of a finite Fourier series with any given accuracy. 


\section{Discussion}

Expanding of scopes of application of classical analytical methods of solving initial boundary value problems is an actual problem. One of the directions of development of such methods is to extend the scope of application of the method of separation of variables - the Fourier method, to problems for areas with curvilinear boundaries. Special functions made it possible to use this method when solving problems for areas with curvilinear boundaries. However, the geometry of such boundaries is connected with coordinate surfaces in some curvilinear coordinate systems, which significantly limits the scope of application of methods. Solutions of initial boundary value problems for regions with complex curvilinear boundaries found using numerical methods do not allow direct analogy with analytical exact solutions in the form of an infinite Fourier series. In this article, a method of separating of variables designed to solve initial boundary value problems for regions with curvilinear boundaries in the general case of their geometry is considered. This method gives solutions in the form of finite generalized Fourier series, which are analogous to partial sums of an infinite Fourier series, which is an exact solution of a problem, and converge to an exact solution. This method pulls together numerical methods for solving initial boundary value problems with an analytical method for solving them. The considered modification of the Fourier method related to orthogonal splines can be used for solving other problems of mathematical physics and continuum mechanics.

\section{Conclusions}

In this method, the potential capabilities of the variable separation method, orthogonal splines, and the finite difference method are used together, which leads to analytical solutions in the form of finite Fourier series that correspond with high accuracy to partial sums of an infinite Fourier series - the exact solution of the problem.

The article [15] shows the effectiveness of orthogonal splines in the problems of approximation of functions and surfaces. Here the region of application of orthogonal splines is expanded.

The method of modeling temperature data using semiparametric additive generalized linear model is proposed in the article [16]. This method can apparently be used in conjunction with the modified Fourier method proposed here in solving initial boundary value problems for temperature fields in regions with a curved boundary.

\section{REFERENCES}

[1] E.A. Gasymov, A.O. Guseinova, U.N. Gasanova
Application of generalized separation of variables to solving mixed problems with irregular boundary conditions // Computational Mathematics and Mathematical Physics, 56:7 (2016), 1305-1309.

[2] I.A. Savichev and A.D. Chernyshov, Application of the angular superposition method to the contact problem on the compression of an elastic cylinder // Mech. Solids, 44:3 (2009), 463-472.

[3] Yu. I. Malov, L.K. Martinson, K.B. Pavlov, Solution by separation of the variables of some mixed boundary value problems in the hydrodynamics of conducting media // Computational Mathematics and Mathematical Physics, 12:3 (1972), 71-86.

[4] M.Sh. Israilov, Diffraction of Acoustic and Elastic Waves on a Half-Plane for Boundary Conditions of Various Types // Mech. Solids, 48:3 (2013), 337-347.

[5] A. Vretblad, Fourier analysis and its applications, Springer-Verlag, New York, Berlin, Heidelber, 2003, 272 p.

[6] A.B. Usov, Finite-difference method for the Navier-Stokes equations in a variable domain with curved boundaries // Computational Mathematics and Mathematical Physics, 48:3 (2008), 464-476.

[7] P.A. Krutitskii, The first initial-boundary value problem for the gravity-inertia wave equation in a multiply connected domain // Computational Mathematics and Mathematical Physics, 37:1 (1997), 113-123.

[8] G. Strang, G.J. Fix, An Analysis of the Finite Element Method. Prentice-Hall, Inc. Englewood Clifs, N. J. 1973. $349 \mathrm{p}$.

[9] V.L. Leontiev, Orthogonal finite functions and numerical methods. Ulyanovsk: Ulyanovsk state university. 2003. 178 p. [In Russian]

[10] V.L. Leont'ev, A variational-grid method involving orthogonal finite functions for solving problems of natural vibrations of 3D elastic solids // Mech. Solids, 37:3 (2002), 101-109.

[11] V.Ya. Arsenin, Methods of Mathematical Physics and Special Functions [in Russian], Nauka, Moscow, 1974, 432 p.

[12] A.A. Samarskii, E. S. Nikolaev, Numerical Methods for Grid Equations. Nauka, Moscow, 1978, 592 p. [in Russian]

[13] A.A. Samarskii, E. S. Nikolaev, Numerical Methods for Grid Equations, vol. I, Birkhäuser Verlag, Basel-Boston-Berlin, 1989, 242 p.

[14] A.A. Samarskii, E. S. Nikolaev, Numerical Methods for Grid Equations, vol. II, Birkhäuser Verlag, Basel-Boston-Berlin. 1989, 502 p.

[15] Leontiev V. L. , "Orthogonal Splines in Approximation of Functions," Mathematics and Statistics, Vol. 8, No. 2, pp. 167 - 172, 2020. DOI: 10.13189/ms.2020.080212.

[16] Azumah Karim , Ananda Omutokoh Kube , Bashiru Imoro Ibn Saeed, "Penalized Maximum Likelihood Estimation of Semiparametric Generalized Linear Models with Application to Climate Temperature Data," Mathematics and Statistics, Vol. 8, No. 4, pp. 472 - 479, 2020. DOI: 10.13189/ms.2020.080415. 